\title{
Constructing three emotion knowledge tests from the invariant measurement approach
}

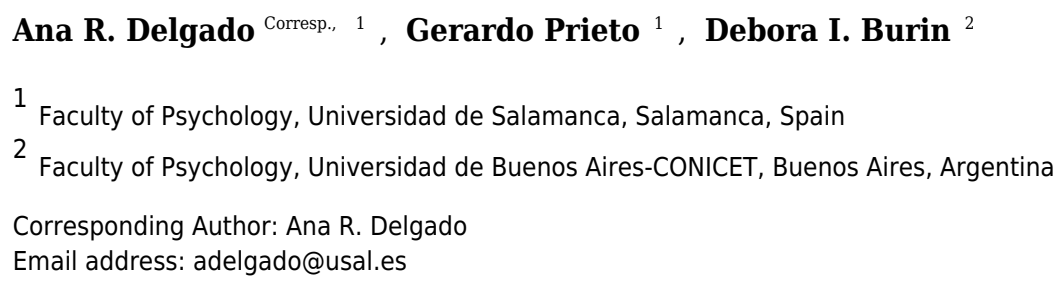

Background. Psychological constructionist models like the Conceptual Act Theory (CAT) postulate that complex states such as emotions are composed of basic psychological ingredients that are more clearly respected by the brain than basic emotions. The objective of this study was the construction and initial validation of Emotion Knowledge measures from the CAT frame by means of an invariant measurement approach, the Rasch Model (RM). Psychological distance theory was used to inform item generation. Methods. Three EK tests -emotion vocabulary (EV), close emotional situations (CES) and far emotional situations (FES)- were constructed and tested with the RM in a community sample of 100 females and 100 males (age range: 18-65), both separately and conjointly. Results. It was corroborated that data-RM fit was good enough. Then the effect of type of test and emotion on Rasch-modelled item difficulty was tested. Significant effects of emotion on EK item difficulty were found, but the only statistically significant difference was that between "happiness" and the remaining emotions; neither type of test, nor interaction effects on EK item difficulty were statistically significant. The testing of gender differences was carried out after corroborating that differential item functioning (DIF) would not be a plausible alternative hypothesis for the results. No statistically significant sex-related differences were found out in EV, CES, FES, or total EK. However, the sign of $d$ indicate that female participants were consistently better than male ones, a result that will be of interest for future meta-analyses. Discussion. The three EK tests are ready to be used as components of a higher-level measurement process. 


\section{Constructing three emotion knowledge tests from the}

\section{3 invariant measurement approach}

4

5 Ana R. Delgado ${ }^{1}$, Gerardo Prieto ${ }^{1}$, Debora I. Burin ${ }^{2}$

$6{ }^{1}$ Faculty of Psychology, Universidad de Salamanca, Salamanca, Spain

$7 \quad{ }^{2}$ Faculty of Psychology, Universidad de Buenos Aires-CONICET, Argentina

8

9 Corresponding Author:

10 Ana R. Delgado ${ }^{1}$

$11{ }^{1}$ Avda. de la Merced 109-131, Salamanca, 37005, Spain

12 Email address: adelgado@usal.es

13

14

15

16

17

18

19

20

21

22

23

24

25

26

27

28

29 
Abstract

31 Background. Psychological constructionist models like the Conceptual Act Theory (CAT)

32 postulate that complex states such as emotions are composed of basic psychological ingredients

33 that are more clearly respected by the brain than basic emotions. The objective of this study was

34 the construction and initial validation of Emotion Knowledge measures from the CAT frame by

35 means of an invariant measurement approach, the Rasch Model (RM). Psychological distance

36 theory was used to inform item generation.

37 Methods. Three EK tests -emotion vocabulary (EV), close emotional situations (CES) and far

38 emotional situations (FES) - were constructed and tested with the RM in a community sample of

39100 females and 100 males (age range: 18-65), both separately and conjointly.

40 Results. It was corroborated that data-RM fit was good enough. Then the effect of type of test

41 and emotion on Rasch-modelled item difficulty was tested. Significant effects of emotion on EK

42 item difficulty were found, but the only statistically significant difference was that between

43 "happiness" and the remaining emotions; neither type of test, nor interaction effects on EK item

44 difficulty were statistically significant. The testing of gender differences was carried out after

45 corroborating that differential item functioning (DIF) would not be a plausible alternative

46 hypothesis for the results. No statistically significant sex-related differences were found out in

$47 \mathrm{EV}, \mathrm{CES}, \mathrm{FES}$, or total EK. However, the sign of $d$ indicate that female participants were

48 consistently better than male ones, a result that will be of interest for future meta-analyses.

49 Discussion. The three EK tests are ready to be used as components of a higher-level

50 measurement process. 


\section{Introduction}

Contrary to the view of emotions as discrete natural events, an amalgam of expression

and behavior with a distinct neural basis, constructionism posits that they are not "ontologically objective" categories or brute facts (Barrett, 2012; Searle, 2010) but ontologically subjective categories. These categories depend on collective intentionality, and not only physical actions and body states. That some of the so-called emotional behaviors (e.g., fight or freeze) have innate circuits does not imply that discrete emotions have them too (Lindquist, Wager, Kober, Bliss-Moreau \& Barrett, 2012). At the neural level, there are no bi-univocal correspondences between a given emotion and areas of activation (Lindquist et al., 2012), and a variety of emotional experiences are associated with dynamic interactions of extended neural networks (Raz et al., 2016).

If we consider emotion categories as ontologically subjective categories, then we can think of them as cognitive tools allowing us to represent the shared meaning of changes in the natural world, i.e., the shared meaning of both internal physical changes and of sensory changes external to the perceiver (Barrett, 2012). Psychological constructionist models such as the Conceptual Act Theory (CAT) postulate that complex states (e.g., emotions and cognitions) are composed of basic psychological ingredients that are brain-based (Barrett, 2009a). The CAT hypothesizes that physical changes are transformed into emotions when taking on psychological functions that require socially shared conceptual knowledge to be meaningful to the perceiver; it is in this sense that emotions are real: they are both biologically evident and part of our social reality (Barrett, 2006, 2012, 2014; Wilson-Mendenhall, Barrett, Simmons, \& Barsalou, 2011). Some emotion categories serve this purpose only for members of one particular culture, but there are some others, e.g., happiness, sadness, anger, fear, and disgust, that can be thought of as closer 
75 to universal and so it is typical to find them in experimental and developmental studies

76 (Lindquist, Gendron, Barrett \& Dickerson, 2014; Tracy \& Randles, 2011).

In any case, emotion categories are not context-independent representations:

78 Emotion knowledge (EK) is situated (Barrett, 2012, 2014; Wilson-Mendenhall, Barrett,

79 Simmons, \& Barsalou, 2011), and thus there are cultural and individual differences in the use of

80 emotion words, a skill that is closely related to emotional intelligence (Barrett, 2009b). Currently,

81 it is not clear whether ability-based emotional intelligence is a construct with the same status as

82 fluid and crystallized intelligence or rather whether it is already defined by extant constructs,

83 such as acculturated knowledge/ crystallized intelligence (MacCann, Joseph, Newman \& Roberts,

84 2014). The predominant operationalization has been the Mayer-Salovey-Caruso Emotional

85 Intelligence Test Battery, whose psychometrical properties are not optimal (Orchard, MacCann,

86 Schulze, Matthews, Zeidner, \& Roberts, 2009). Given that emotional aptitude variables predict

87 dependent variables as relevant as perceived stress (Rey, Extremera \& Pena, 2016) or depressive

88 symptoms (Luque-Reca, Augusto-Landa \& Pulido-Martos, 2016) we should start to test

89 narrowly defined emotion domains each requiring its own theories and measures (Matthews,

90 Zeidner \& Roberts, 2012).

The fact that both categorical knowledge and contextual information are constitutive of

92 emotions is a substantive reason to prefer invariant measurement models over the reflective

93 structural equation models usually employed in validation studies; there are also psychometric

94 reasons (e.g., Engelhard \& Wang, 2014) to prefer invariant measurement models to the formative

95 structural equation models recommended by Coan and Gonzalez (2015) in the CAT context. An

96 implementation of the invariant measurement approach is the Rasch model (RM; Rasch, 1960),

97 increasingly used to validate psychological and neuropsychological tests (Delgado, 2012; 
98 Engelhard \& Wang, 2014; Miguel, Silva, \& Prieto, 2013; Prieto, Delgado, Perea \& Ladera,

99 2010). The probability that subject $n$ passes item $i$ is modeled as Pni $=\exp (\mathrm{Bn}-\mathrm{Di}) /(1+\exp [\mathrm{Bn}-$

$100 \mathrm{Di}]$ ), where $\mathrm{Bn}$ is the person level and $\mathrm{Di}$ is the item location. This logistic one-parameter model

101 shows the property of specific objectivity, allowing the algebraic separation of items and person

102 parameters (the person parameter can be erased when estimating the item parameters). This is so

103 because the sum score for an item or person is a sufficient statistic for the corresponding

104 parameter, i.e., it captures all the information about the corresponding parameter that is

105 contained in the sample.

106 One of the main advantages of the RM derives from the fact that it is a conjoint

107 measurement model: If empirical data fit the model adequately, then person measures (e.g.,

108 aptitude, personality trait) and item locations (e.g., difficulty, severity) can be jointly located on

109 an interval scale (variable map) whose unit is the logit. When using the RM, item parameter

110 estimations are sample-independent and person parameter estimations are independent of the

111 particular items that have been used; this is not true of the classical measurement model. Of great

112 interest for psychological measurement is the fact that the level of analysis is the individual in

113 the RM, while Structural Equation Models (which are statistical models for covariances), use the

114 group as level of analysis (Engelhard \& Wang, 2014).

115 Thus, the general objective of this study was the construction and initial validation of EK

116 measures from a psychological constructionist theoretical frame, the CAT, by means of an

117 invariant measurement approach, the RM. When validating situational tests of emotion

118 understanding, it has been found that items describing situations with close/concrete receivers

119 are easier than those in which receivers are far/abstract (Delgado, 2016), a result that is predicted

120 by psychological distance theories (Soderberg, Callahan, Kochersberger, Amit \& Ledgerwood, 
121 2015;Trope \& Liberman, 2010), and so the close/far distinction has been taken into account in

122 the generation of items for the situational tests. Three EK tests have been constructed and tested

123 with the RM, both separately -vocabulary, close and far situations- and conjointly, given that

124 they are all EK measures.

125

\section{Materials \& Methods}

\section{Participants}

The sample was composed of 100 females and 100 males, with ages ranging from 18 to

65 years old, Spanish as first language, and Spanish nationality. Roughly half of them (n=94)

were young adults (18-30). The educational level was high (155 participants were or had been to college or further).

Even though the property of specific objectivity allows the person-independent estimation of item parameters, i.e., no representative sample is needed, we obtained the most heterogeneous sample that was available to us by recruiting participants from various Spanish regions in an art museum that was public and open to all.

\section{Instruments}

Three tests were constructed with LiveCode 4.6 (2011) and implemented on a portable computer. Identification, gender, age, informed consent, response option and right/wrong answers were asked for and automatically stored by the application. Each of the 3 tests was composed of 40 multiple-choice items, 8 for each of the 5 emotion "families" of happiness,

140 sadness, anger, fear, and disgust. There was no time limit, and feedback on the total score

141 (number of correct answers; possible range: 0-120) was provided in the last screen. Tests are

142 described below in the order they were applied. Item examples can be seen in Figure 1. 
144 from the corpus of the Royal Spanish Academy CORPES XXI, which has 25 million of forms

145 for each year between 2001 and 2012 (Real Academia Española, 2014). The five response

146 options, of which only one is correct, were happiness, sadness, anger, fear, and disgust (alegría,

147 tristeza, ira, miedo, and asco, in standard Spanish). The subject had to choose the response

148 option whose meaning was the closest to that of the target word.

149 Close Emotional Situations (CES). Item stems were verbal scenarios showing a

150 character and a close/concrete moment, act, object and place. Scenarios described concrete

151 variations of the prototypes of the five emotion "families". Some 40 first names (half of them

152 male) were selected from the database of the National Statistical Institute so that each scenario

153 showed a different character identified by his/her name. There were five response options:

154 happiness, sadness, anger, fear, and disgust. This is the adequate level of specificity for this kind

155 of test, given that it has been found that emotional inferences from verbal scenarios are more

156 specific than valence and class, but not more specific than emotion "families" (Molinari et al.,

157 2009). The subject had to choose the option that best described the emotion that would be typical

158 to feel in that concrete situation.

159 Far Emotional Situations (FES). Item stems were verbal scenarios showing a

160 far/abstract time, character and situation. Scenarios described abstract variations of the

161 prototypes of the five emotion "families" and the main character was not identified by his/her

162 first name but by a generic label (half of them female, e.g., "a girl"). Response options were the

163 same as in the previous tests, and the task was to choose the one that best described the emotion

164 that would usually be felt in that abstract situation. 

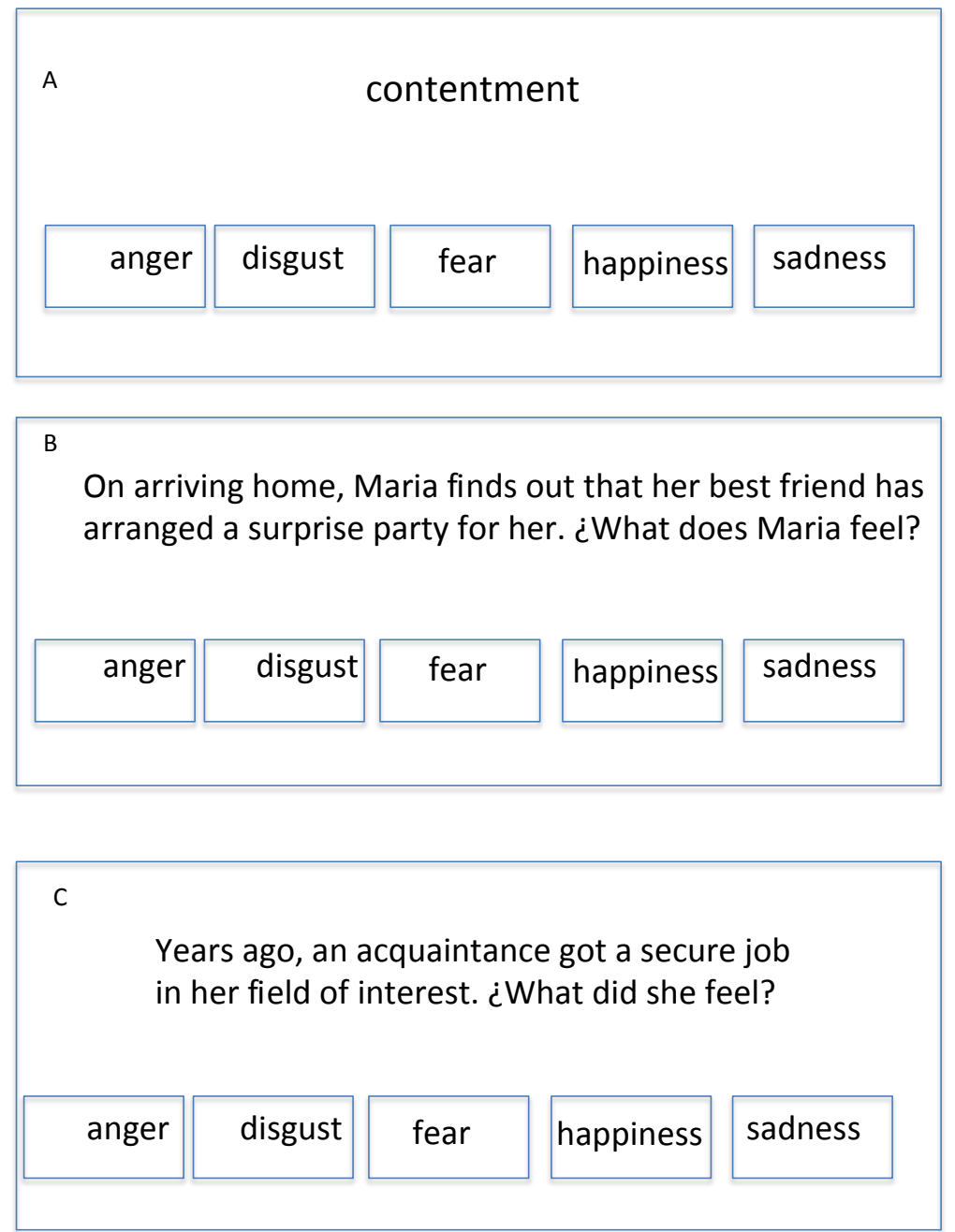

167 Figure 1.

168 Three HAPPINESS item examples: (A) EV item. (B) CES item. (C) FES item.

169 Note: Items were written in Spanish, so the translation is an approximation.

\section{Procedure}

171 Participants were approached by a university researcher with a visible identification card,

172 and asked about age, provenance and first language to warrant inclusion criteria. After asking for

173 consent to use the data for research purposes, the tests were individually applied on a portable

174 computer. 


\section{Data Analysis}

176 Responses to the three tests were separately analyzed with the RM. Then, after conjoint

177 scaling of items and persons, the effect of type of test and emotion on item difficulty was probed

178 by means of factorial ANOVA.

179 Rasch analyses were performed with the computer program Winsteps 3.80.1 (Linacre,

180 2013). Data-model fit is assessed by outfit (calculated by adding the standardized square of

181 residuals after fitting the model over items or subjects to form chi-square-distributed variables)

182 and infit (an information-weighted form of outfit). Infit /outfit values over 2 distort the

183 measurement system (Linacre, 2013). Unidimensionality is a requirement for the model, not

184 implying that performance is due to a unique psychological process (Reckase, 1979); component

185 analyses of residuals are performed by Winsteps 3.80 .1 in order to test this assumption. The

186 indications are that Rasch measures should account for at least $20 \%$ of the total variance and it is

187 recommended that the unexplained variance in the first contrast be lower than 3 (Miguel, Silva \& 188 Prieto, 2013).

189 Differential item functioning (DIF) analysis tests the generalized validity of the measures

190 for different groups. In this case, given that there is evidence of female superiority in the

191 accuracy of affective judgements (Hall, Gunnery \& Horgan, 2016), a plausible alternative

192 hypothesis is the instrumental one: items could be functioning differently for males and females.

193 Thus, the DIF hypothesis was tested: The standardized difference between item calibrations in

194 the case of two groups (i.e., male and female) was calculated and tested using Bonferroni-

195 corrected alpha levels; the Rasch aptitude estimates from the analysis of all the data were held

196 constant, providing the conjoint measurement scale in logit units (Linacre, 2013).

197

\section{Results}




\section{EV test}

199 One person and two items got extreme scores (i.e., zero or perfect raw scores) and so

200 their Rasch measures were not estimated. The Rasch analysis of the remaining data indicates

201 good data-model fit for items, mean infit was .99 $(S D=.08)$ and mean outfit was .91 $(S D=.31)$.

202 For persons, mean infit was $1.00(S D=.25)$ and mean outfit was .91 $(S D=.64)$. No item

203 showed infit/outfit over 1.5. Twelve persons (6\%) showed outfit over 2, but just one of them

204 showed infit over 2 . The percentage of variance explained by EV measures was $33.9 \%$ and the

205 component analysis of residuals showed that the unexplained variance in the first contrast was

206 2.3. Finally, item reliability (.96) and model person reliability (.72) were good enough. Table 1

207 shows the main results of the item analysis.

208 Table 1.

209 Emotion Vocabulary Items: Rasch Analysis Results.

210

\begin{tabular}{lrrrrr}
\hline Item & Score & Rasch $D i$ & \multicolumn{1}{l}{$S E$} & Infit & Outfit \\
01 & 198 & -2.95 & .72 & 1.01 & 1.13 \\
02 & 137 & 1.26 & .17 & .94 & .90 \\
03 & 185 & -.75 & .28 & .88 & .61 \\
04 & 194 & -1.79 & .43 & .93 & .35 \\
05 & 194 & -1.79 & .43 & .91 & .86 \\
06 & 158 & .60 & .19 & .99 & 1.01 \\
07 & 199 & -3.66 & 1.01 & .98 & .20 \\
08 & 190 & -1.22 & .34 & .92 & .53 \\
09 & 162 & .46 & .20 & 1.05 & .99 \\
10 & 148 & .94 & .18 & 1.04 & 1.00 \\
11 & 193 & -1.62 & .40 & .82 & .32 \\
12 & 200 & --- & --- & --- & --- \\
13 & 193 & -1.62 & .40 & .95 & .44 \\
14 & 189 & -1.11 & 32 & .98 & .62 \\
15 & 164 & .38 & .20 & 1.04 & .99 \\
16 & 155 & .71 & .18 & .98 & .88 \\
17 & 173 & -.02 & .22 & 1.02 & .97 \\
18 & 200 & --- & --- & --- & --- \\
19 & 165 & .34 & .20 & 1.05 & 1.19 \\
20 & 186 & -.83 & .29 & 1.03 & .73 \\
21 & 75 & 2.84 & .16 & 1.06 & 1.27 \\
22 & 147 & .97 & .18 & .91 & .97 \\
\hline & & & & &
\end{tabular}




\begin{tabular}{rrrrrr}
\hline 23 & 95 & 2.34 & .16 & .96 & .99 \\
24 & 133 & 1.37 & .17 & 1.04 & .96 \\
25 & 171 & .08 & .22 & 1.13 & 1.22 \\
26 & 157 & .64 & .19 & .92 & .89 \\
27 & 192 & -1.47 & .37 & .87 & .37 \\
28 & 171 & .08 & .22 & .89 & .74 \\
29 & 172 & .03 & .22 & 1.01 & .87 \\
30 & 182 & -.53 & .26 & 1.11 & 1.50 \\
31 & 111 & 1.94 & .16 & 1.23 & 1.43 \\
32 & 196 & -2.23 & .52 & 1.04 & 1.07 \\
33 & 170 & .13 & .21 & 1.01 & .87 \\
34 & 52 & 3.49 & .18 & 1.01 & 1.03 \\
35 & 177 & -.22 & .24 & .96 & .91 \\
36 & 175 & -.12 & .23 & .92 & 1.19 \\
37 & 176 & -.17 & .23 & 1.02 & 1.10 \\
38 & 190 & -1.22 & .34 & 1.04 & .96 \\
39 & 53 & 3.46 & .18 & 1.02 & 1.25 \\
40 & 137 & 1.26 & .17 & 1.00 & .95 \\
\hline
\end{tabular}

Average person aptitude in logit units was $2.19, S D=1.04$, range $=-1.12$ to 4.78 . Just one

213 item (EV24, happiness) showed sex-related DIF favoring male subjects, i.e., male subjects had a

214 higher probability of passing this item than female subjects with the same total score. No gender

215 differences (impact) in Rasch measures were found, Welch- $t(196)=.68, p=.50, d=-.11$

216 (conventionally, $0=$ female, $1=$ male). As an illustration, Table 2 shows the map of the variable,

217 where the right side shows item locations while person measures are situated at the left.

218 Table 2.

219 Emotion Vocabulary-Variable Map.

220

221

222

223

224

225

226

227

228

229

230

231

232

233

234

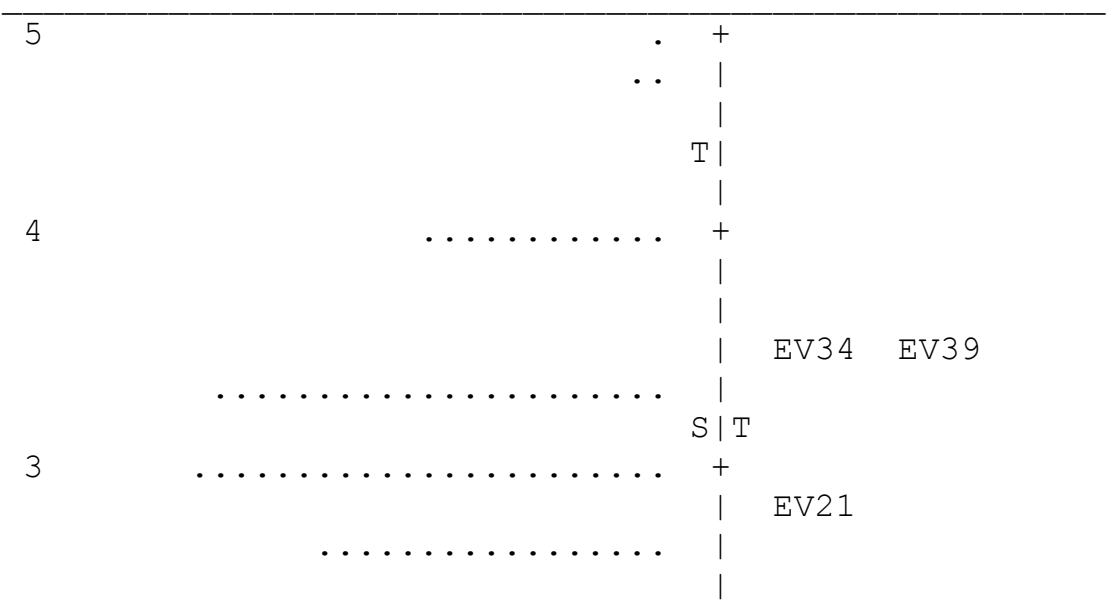


236

237

238

239

240

241

242

243

244

245

246

247

248

249

250

251

252

253

254

255

256

257

258

259

260

261

262

263

264

265

266

267

$268-3$

269

270

271

272

273

274

275

276

277

278

279

280

$-4$

Note. $\mathrm{M}=$ mean; $\mathrm{S}=1 S D ; \mathrm{T}=2 S D$.

\section{CES test}

Seven persons obtained extreme scores; their Rasch measures were not estimated. The

Rasch analysis indicates good data-model fit: Item mean infit $=.98(S D=.11)$, mean outfit $=.87$

$281(S D=.25)$; person mean infit $=1.00(S D=.17)$, mean outfit $=.87(S D=.74)$. No item showed

282 infit/outfit over 2. Eight persons (4\%) showed outfit over 2. The percentage of variance explained

283 by close emotional situations measures was $22.9 \%$ and the component analysis of residuals

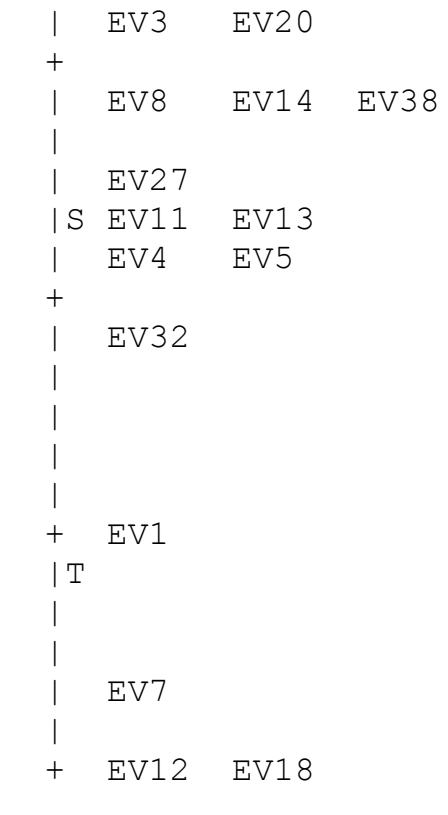


284 showed that the unexplained variance in the first contrast was 2.3. As to item reliability and 285 model person reliability, they were .93 and .58 , respectively. Table 3 shows the main results of 286 the item analysis.

287 Table 3.

288 Close Emotional Situations Items: Rasch Analysis Results. 289

\begin{tabular}{lrrrrr}
\hline Item & Score & Rasch $D i$ & \multicolumn{1}{l}{ SE } & \multicolumn{1}{l}{ Infit } & Outfit \\
01 & 148 & 1.51 & .17 & 1.16 & 1.32 \\
02 & 190 & -.65 & .34 & 1.06 & .74 \\
03 & 197 & -2.01 & .61 & 1.07 & .64 \\
04 & 169 & .76 & .21 & 1.04 & 1.09 \\
05 & 197 & -2.01 & .61 & .80 & .37 \\
06 & 123 & 2.19 & .16 & 1.13 & 1.22 \\
07 & 138 & 1.80 & .17 & 1.08 & 1.03 \\
08 & 107 & 2.59 & .16 & 1.07 & 1.06 \\
09 & 197 & -2.01 & .61 & .87 & .83 \\
10 & 198 & -2.45 & .73 & .80 & .80 \\
11 & 183 & -.01 & .27 & .94 & .73 \\
12 & 188 & -.43 & .31 & .89 & .57 \\
13 & 164 & .96 & .20 & 1.07 & .96 \\
14 & 160 & 1.11 & .19 & .97 & .96 \\
15 & 187 & -.34 & .30 & 1.02 & .87 \\
16 & 139 & 1.77 & .17 & .92 & $.89-$ \\
17 & 195 & -1.44 & .47 & .88 & .66 \\
18 & 157 & 1.22 & .19 & .90 & .81 \\
19 & 161 & 1.08 & .19 & 1.16 & 1.66 \\
20 & 179 & .25 & .25 & 1.03 & .77 \\
21 & 182 & .06 & .26 & 1.06 & .98 \\
22 & 196 & -1.69 & .53 & .74 & .44 \\
23 & 180 & .19 & .25 & .97 & .68 \\
24 & 176 & .42 & .23 & 1.04 & 1.00 \\
25 & 189 & -.54 & .33 & .97 & .83 \\
26 & 191 & -.77 & .36 & 1.17 & .98 \\
27 & 195 & -1.44 & .47 & 1.15 & .73 \\
28 & 187 & -.34 & .30 & .92 & .62 \\
29 & 185 & -.17 & .28 & .89 & .56 \\
30 & 166 & .88 & .20 & .97 & .82 \\
31 & 190 & -.65 & .34 & .99 & 1.13 \\
32 & 198 & -2.45 & .73 & .81 & 1.07 \\
33 & 189 & -.54 & .33 & .87 & .61 \\
34 & 191 & -.77 & .36 & 1.01 & .89 \\
35 & 192 & -.91 & .38 & .86 & .76 \\
36 & 173 & .57 & .22 & 1.06 & 1.11 \\
\hline & & & & &
\end{tabular}




\begin{tabular}{rrrrrr}
\hline 37 & 121 & 2.24 & .16 & .99 & .94 \\
38 & 159 & 1.15 & .19 & 1.09 & 1.19 \\
39 & 194 & -1.24 & .44 & .90 & .55 \\
40 & 126 & 2.11 & .16 & .99 & .99 \\
\hline
\end{tabular}

291

Average person aptitude in logit units was $2.66, S D=.93$, range $=-1.68$ to 4.45 . Neither

292 sex-related DIF nor gender significant differences were found, Welch- $t(182)=1.39, p=.17, d=$ 293 .21 .

FES test

Four persons got extreme scores. The Rasch analysis of the remaining data indicates good 296 data-model fit: For items, mean infit was $.97(S D=.11)$ and mean outfit was .88 $(S D=.32)$. For 297 persons, mean infit was $1.00(S D=.18)$ and mean outfit was $.88(S D=.59)$. No item showed 298 infit /oufit over 2; eight persons showed outfit over 2. The percentage of variance explained by 299 measures was $22.9 \%$ and the component analysis of residuals showed that the unexplained 300 variance in the first contrast was 3.2. Finally, item reliability (.93) and model person reliability 301 (.65) were acceptable. Table 4 shows the main results of the item analysis.

302 Table 4.

303 Far Emotional Situations Items: Rasch Analysis Results. 304

\begin{tabular}{rrrrrr}
\hline Item & Score & Rasch $D i$ & \multicolumn{1}{l}{ SE } & \multicolumn{1}{l}{ Infit } & Outfit \\
01 & 184 & -.35 & .28 & 1.19 & .95 \\
02 & 160 & .87 & .19 & .97 & .89 \\
03 & 178 & .06 & .24 & 1.14 & 1.41 \\
04 & 144 & 1.39 & .17 & 1.09 & 1.25 \\
05 & 195 & -1.76 & .49 & .79 & .98 \\
06 & 170 & .47 & .21 & 1.04 & 1.15 \\
07 & 166 & .64 & .20 & 1.02 & 1.01 \\
08 & 171 & .42 & .22 & 1.01 & .94 \\
09 & 161 & .83 & .19 & 1.00 & .94 \\
10 & 198 & -2.83 & .75 & .79 & .32 \\
11 & 177 & .11 & .24 & 1.03 & .90 \\
12 & 160 & .87 & .19 & .96 & 1.02 \\
13 & 196 & -2.02 & .54 & .87 & .26 \\
14 & 190 & -.93 & .35 & .95 & 1.47 \\
15 & 164 & .72 & .20 & 1.06 & .98 \\
\hline
\end{tabular}




\begin{tabular}{rrrrrr}
\hline 16 & 176 & .17 & .23 & .98 & .86 \\
17 & 115 & 2.15 & .16 & .97 & 1.13 \\
18 & 175 & .22 & .23 & .97 & .78 \\
19 & 142 & 1.44 & .17 & 1.02 & 1.02 \\
20 & 191 & -1.05 & .37 & .85 & .46 \\
21 & 82 & 2.96 & .16 & 1.17 & 1.20 \\
22 & 171 & .42 & .22 & 1.04 & 1.15 \\
23 & 184 & -.35 & .28 & .91 & .58 \\
24 & 158 & .94 & .19 & .97 & 1.14 \\
25 & 192 & -1.20 & .39 & .83 & .44 \\
26 & 175 & .22 & .23 & .95 & .68 \\
27 & 195 & -1.76 & .49 & .71 & .21 \\
28 & 171 & .42 & .22 & .95 & .81 \\
29 & 192 & -1.20 & .39 & 1.02 & .81 \\
30 & 192 & -1.20 & .39 & .85 & .64 \\
31 & 145 & 1.36 & .17 & 1.09 & 1.06 \\
32 & 189 & -.81 & .33 & .85 & .52 \\
33 & 194 & -1.54 & .45 & .82 & .53 \\
34 & 185 & -.43 & .29 & .97 & .74 \\
35 & 122 & 1.98 & .16 & 1.24 & 1.69 \\
36 & 194 & -1.54 & .45 & .81 & .73 \\
37 & 168 & .55 & .21 & 1.07 & 1.10 \\
38 & 165 & .68 & .20 & .97 & .92 \\
39 & 185 & -.43 & .29 & .97 & .88 \\
40 & 186 & -.51 & .30 & .95 & .56 \\
\hline & & & & &
\end{tabular}

305

306

Average person aptitude in logit units was $2.46, S D=1.09$, range $=-2.44$ to 4.34 . No item

307 showed sex-related DIF. No gender differences in Rasch measures were found Welch-t $(183)=$

$308.14, p=.89, d=-.02$ (conventionally, code was $0=$ female, $1=$ male).

309

310 Total EK score

311 Two items had extreme scores and so their Rasch scores were not estimated. The Rasch

312 analysis of the responses to the remaining 118 items indicates good data-model fit: for items,

313 mean infit was $.99(S D=.07)$ and mean outfit was $.90(S D=.25)$. For persons, mean infit was 1.00

$314(S D=.14)$ and mean outfit was $.90(S D=.42)$. No item showed infit/outfit over 2 , and just six

315 persons out of 200 showed outfit over 2 . The percentage of variance explained by EK measures 
316 was $22.7 \%$ and the component analysis of residuals showed that the unexplained variance in the

317 first contrast was 4.5 (2.9\%). Finally, item reliability (.94) and model person reliability (.83)

318 were good. Table 5 shows the main results of the item analysis.

319 Table 5.

320 Emotion Knowledge Items: Rasch Analysis Results.

321

\begin{tabular}{llrrrrr}
\hline Item & EMOTION & Score & Rasch Di & \multicolumn{1}{l}{ SE } & \multicolumn{1}{l}{ Infit } & Outfit \\
001 & HAPPINESS & 198 & -2.59 & .72 & 1.05 & 1.25 \\
002 & DISGUST & 137 & 1.48 & .16 & .98 & 1.01 \\
003 & ANGER & 185 & -.41 & .28 & .96 & .70 \\
004 & FEAR & 194 & -1.43 & .42 & .87 & .53 \\
005 & DISGUST & 194 & -1.43 & .42 & 1.00 & 1.13 \\
006 & DISGUST & 158 & .87 & .18 & .98 & .94 \\
007 & HAPPINESS & 199 & -3.30 & 1.01 & 1.02 & .72 \\
008 & SADNESS & 190 & -.87 & .33 & .92 & .76 \\
009 & ANGER & 162 & .74 & .19 & 1.04 & 1.01 \\
010 & DISGUST & 148 & 1.18 & .17 & .99 & .96 \\
011 & SADNESS & 193 & -1.27 & .39 & .96 & .44 \\
012 & HAPPINESS & 200 & -- & -- & -- & -- \\
013 & HAPPINESS & 193 & -1.27 & .39 & 1.00 & .61 \\
014 & SADNESS & 189 & -.77 & .32 & 1.05 & .94 \\
015 & DISGUST & 164 & .66 & .19 & 1.00 & .97 \\
016 & FEAR & 155 & .97 & .18 & .98 & .94 \\
017 & FEAR & 173 & .29 & .22 & 1.00 & 1.10 \\
018 & HAPPINESS & 200 & -- & -- & -- & -- \\
019 & ANGER & 165 & .63 & .19 & 1.03 & 1.02 \\
020 & HAPPINESS & 186 & -.49 & .29 & 1.09 & 1.07 \\
021 & FEAR & 75 & 2.91 & .15 & 1.12 & 1.14 \\
022 & ANGER & 147 & 1.21 & .17 & .96 & .88 \\
023 & DISGUST & 95 & 2.46 & .15 & 1.01 & .98 \\
024 & HAPPINESS & 133 & 1.58 & .16 & 1.04 & 1.09 \\
025 & FEAR & 171 & .38 & .21 & 1.06 & 1.14 \\
026 & SADNESS & 157 & .91 & .18 & .98 & .99 \\
027 & ANGER & 192 & -1.12 & .37 & 1.00 & .48 \\
028 & SADNESS & 171 & .38 & .21 & .97 & .86 \\
029 & HAPPINESS & 172 & .34 & .21 & 1.03 & .95 \\
030 & DISGUST & 182 & -.20 & .26 & 1.09 & .99 \\
031 & FEAR & 111 & 2.10 & .15 & 1.18 & 1.30 \\
032 & FEAR & 196 & -1.87 & .51 & 1.04 & .93 \\
033 & SADNESS & 170 & .42 & .21 & .99 & 1.02 \\
034 & DISGUST & 52 & 3.50 & .17 & 1.02 & 1.01 \\
035 & ANGER & 177 & .09 & .23 & 1.04 & 1.03 \\
036 & SADNESS & 175 & .20 & .22 & .96 & .85 \\
\hline & & & & & &
\end{tabular}




\begin{tabular}{llrrrrr}
\hline 037 & SADNESS & 176 & .14 & .23 & 1.04 & 1.08 \\
038 & FEAR & 190 & -.87 & .33 & 1.03 & .90 \\
039 & ANGER & 53 & 3.47 & .17 & 1.12 & 1.31 \\
040 & ANGER & 137 & 1.48 & .16 & 1.03 & 1.01 \\
041 & ANGER & 148 & 1.18 & .17 & 1.12 & 1.19 \\
042 & FEAR & 190 & -.87 & .33 & 1.03 & .86 \\
043 & FEAR & 197 & -2.17 & .59 & 1.03 & 1.00 \\
044 & FEAR & 169 & .47 & .20 & .99 & .98 \\
045 & HAPPINESS & 197 & -2.17 & .59 & .85 & .29 \\
046 & ANGER & 123 & 1.82 & .15 & 1.07 & 1.08 \\
047 & SADNESS & 138 & 1.45 & .16 & 1.10 & 1.09 \\
048 & DISGUST & 107 & 2.19 & .15 & 1.10 & 1.10 \\
049 & HAPPINESS & 197 & -2.17 & .59 & .90 & 1.05 \\
050 & HAPPINESS & 198 & -2.59 & .72 & .87 & .61 \\
051 & FEAR & 183 & -.27 & .26 & .96 & .90 \\
052 & SADNESS & 188 & -.67 & .31 & .89 & .67 \\
053 & ANGER & 164 & .66 & .19 & 1.03 & 1.00 \\
054 & ANGER & 160 & .80 & .19 & .99 & .94 \\
055 & ANGER & 187 & -.58 & .30 & 1.01 & .92 \\
056 & DISGUST & 139 & 1.43 & .16 & 1.04 & 1.03 \\
057 & HAPPINESS & 195 & -1.63 & .46 & .87 & .39 \\
058 & DISGUST & 157 & .91 & .18 & 1.02 & 1.02 \\
059 & SADNESS & 161 & .77 & .19 & 1.11 & 1.60 \\
060 & DISGUST & 179 & -.02 & .24 & 1.06 & 1.06 \\
061 & ANGER & 182 & -.20 & .26 & .97 & .87 \\
062 & HAPPINESS & 196 & -1.87 & .51 & .82 & .39 \\
063 & SADNESS & 180 & -.08 & .24 & .97 & .78 \\
064 & FEAR & 176 & .14 & .23 & .93 & .79 \\
065 & FEAR & 189 & -.77 & .32 & .95 & .75 \\
066 & DISGUST & 191 & -.99 & .35 & 1.08 & .93 \\
067 & FEAR & 195 & -1.63 & .46 & 1.05 & .86 \\
068 & ANGER & 187 & -.58 & .30 & .96 & .82 \\
069 & DISGUST & 185 & -.41 & .28 & .93 & .70 \\
070 & SADNESS & 166 & .59 & .20 & 1.02 & .97 \\
071 & ANGER & 190 & -.87 & .33 & .99 & 1.37 \\
072 & HAPPINESS & 198 & -2.59 & .72 & .87 & 1.43 \\
073 & SADNESS & 189 & -.77 & .32 & .89 & .74 \\
074 & FEAR & 191 & -.99 & .35 & .98 & 1.05 \\
075 & HAPPINESS & 192 & -1.12 & .37 & .88 & .76 \\
076 & DISGUST & 173 & .29 & .22 & 1.05 & 1.03 \\
077 & SADNESS & 121 & 1.87 & .15 & 1.04 & 1.02 \\
078 & SADNESS & 159 & .84 & .18 & 1.03 & 1.06 \\
079 & DAPPINESS & 194 & -1.43 & .42 & .90 & .78 \\
& DISGUST & 126 & 1.75 & .15 & .98 & .95 \\
0 & 184 & -.34 & .27 & 1.07 & 1.12 \\
0 & 160 & .80 & .19 & 1.02 & 1.05 \\
\hline
\end{tabular}




\begin{tabular}{llrrrrr}
\hline 083 & FEAR & 178 & .04 & .23 & 1.04 & 1.10 \\
084 & SADNESS & 144 & 1.29 & .17 & 1.03 & 1.04 \\
085 & HAPPINESS & 195 & -1.63 & .46 & .89 & .77 \\
086 & ANGER & 170 & .42 & .21 & .97 & .88 \\
087 & ANGER & 166 & .59 & .20 & 1.02 & 1.05 \\
088 & DISGUST & 171 & .38 & .21 & 1.09 & 1.19 \\
089 & SADNESS & 161 & .77 & .19 & 1.00 & 1.01 \\
090 & HAPPINESS & 198 & -2.59 & .72 & .84 & .19 \\
091 & DISGUST & 177 & .09 & .23 & 1.02 & .94 \\
092 & FEAR & 160 & .80 & .19 & .96 & .88 \\
093 & HAPPINESS & 196 & -1.87 & .51 & .85 & .29 \\
094 & HAPPINESS & 190 & -.87 & .33 & .96 & 1.19 \\
095 & SADNESS & 164 & .66 & .19 & 1.04 & .99 \\
096 & ANGER & 176 & .14 & .23 & .97 & .89 \\
097 & SADNESS & 115 & 2.01 & .15 & 1.02 & 1.03 \\
098 & SADNESS & 175 & .20 & .22 & .97 & .82 \\
099 & DISGUST & 142 & 1.35 & .16 & .98 & .96 \\
100 & DISGUST & 191 & -.99 & .35 & .91 & .55 \\
101 & FEAR & 82 & 2.75 & .15 & 1.11 & 1.18 \\
102 & FEAR & 171 & .38 & .21 & 1.04 & 1.16 \\
103 & SADNESS & 184 & -.34 & .27 & .92 & .69 \\
104 & ANGER & 158 & .87 & .18 & .98 & .91 \\
105 & FEAR & 192 & -1.12 & .37 & .93 & .57 \\
106 & FEAR & 175 & .20 & .22 & .90 & .70 \\
107 & HAPPINESS & 195 & -1.63 & .46 & .85 & .29 \\
108 & DISGUST & 171 & .38 & .21 & .95 & .88 \\
109 & SADNESS & 192 & -1.12 & .37 & .96 & .94 \\
110 & HAPPINESS & 192 & -1.12 & .37 & .90 & .47 \\
111 & ANGER & 145 & 1.26 & .17 & 1.06 & 1.06 \\
112 & FEAR & 189 & -.77 & .32 & .88 & .54 \\
113 & HAPPINESS & 194 & -1.43 & .42 & .82 & .38 \\
114 & SADNESS & 185 & -.41 & .28 & .92 & .69 \\
115 & DISGUST & 122 & 1.84 & .15 & 1.20 & 1.28 \\
116 & HAPPINESS & 194 & -1.43 & .42 & .91 & .55 \\
117 & ANGER & 168 & .51 & .20 & 1.01 & .95 \\
118 & ANGER & 165 & .63 & .19 & .96 & .90 \\
119 & FEAR & 185 & -.41 & .28 & .96 & .80 \\
120 & DISGUST & 186 & -.49 & .29 & .86 & .56 \\
\hline & & & & & &
\end{tabular}

Average person aptitude in logit units was $2.34, S D=.75$, range $=-1.11$ to 4.91 . No item

324 showed sex-related DIF; significant gender differences (impact) in Rasch measures were not

325 found Welch-t $(193)=1.18, p=.24, d=-.17$ (conventionally, code was $0=$ female, $1=$ male). 

order) was -1.54 (HAPPINESS), -0.07 (FEAR), 0.26 (SADNESS), 0.56 (ANGER), and 0.66 (DISGUST). A factorial ANOVA of the effects of type of test and emotion on EK item difficulty was statistically significant, $F(14,103)=5.09, p<.001$. Neither type of test, $F(2$, $103)=1.78, p=.17$, nor the interaction effects $F(8,103)=1.06, p=.40$, were statistically significant. Emotion effects on EK item difficulty were found, $F(4,103)=14.02, p=p<.001$; Bonferroni post hoc tests indicated that the only statistically significant difference was that

334 between HAPPINESS and the remaining emotions.

\section{Discussion} constructionist theoretical frame: one vocabulary and two situational tests. Although items were generated in Spanish, the careful selection of the emotion words and the substantive background (conceptual act and psychological distance theories) should facilitate their adaptation to other languages and/or cultures. Test items and specifications will be made available upon request to accredited researchers for non-commercial purposes.

344 three tests separately -EV, CES and FES- and conjointly, given that all the items were designed

345 to provide EK measures. In the four cases, data-model fit was good enough, so the probability of

346 a response can be expressed as an additive function of a person parameter and an item parameter;

347 this is consistent with the quantitative assumption implicitly made - but not tested-in most

348 psychological assessment situations (Michell, 1999). Even though the first contrast of the 
349 component analysis of residuals was slightly over the recommended value for one of the tests, as

350 well as for the conjoint scaling of the three tests, some evidence of multidimensionality should

351 be expected when measuring complex constructs, e.g., when measuring math ability, some

352 evidence of multidimensionality is better tolerated than when measuring geometry aptitude

353 (Linacre, 2013).

354 It is relevant to note here that the use of parametric statistical methods takes for granted

355 interval status, even though the nature of many scoring systems is ordinal at best. We have

356 evaluated the interval scaling assumption with the RM, which because of its desirable metric

357 properties can be used to quantify different types of experimental data (Delgado, 2007). Some

358 other advantages of the RM, at the practical level, are the ease of interpreting and

359 communicating results: because both participants and items are located on the same variable,

360 comparisons can be made concerning what items have been passed by what persons (Prieto,

361 Delgado, Perea \& Ladera, 2010).

362

As to gender differences, at least three quantitative reviews have shown clear evidence of

363 female superiority in the accuracy of affective judgments; effect sizes are small-to-medium

364 following conventional standards (Hall, Gunnery \& Horgan, 2016). In our study, the testing of

365 gender differences was carried out after corroborating that item DIF would not be a plausible

366 alternative hypothesis for the results. No statistically significant sex-related differences were

367 found in EV, CES, FESA or total EK score. However, the sign of $d$ indicate that female

368 participants scored consistently higher than male ones, -.11 (EV), -0.21 (CES), -0.02 (FES) and

$369-.17$ (total EK), a result that will be of interest for future meta-analyses. For instance, a recent

370 multi-level meta-analysis by Thompson and Voyer (2014) found that the effect size of the

371 difference in emotion perception, a basic emotional aptitude, is $d=-.19$ (if coded as female $=0$, 
372 male=1), not far from the $d=-.17$ found on our study for the EK measures. Given such a small

373 effect size, finding statistically significant sex-related differences in EK would require studies

374 with very large samples.

375 Finally, when EK items from the three tests were conjointly scaled, item difficulty did not

376 statistically differ as a function of the original test (if CES, FES or EV, ordered by ascending

377 average item difficulty) and so they could be used somewhat interchangeably when measuring

378 EK with time restrictions. This is not implying that the three tests are measuring the same

379 processes (in an essentialist way), only that there is one latent variable (EK), all items tap into it,

380 and the level of this EK variable is in a certain moment the focus of measurement interest $(\mathrm{Wu}$,

381 Tam, \& Jen, 2017). Descriptively, the average item difficulty was ordered as expected from

382 psychological distance theories: CES item scenarios were designed as the most concrete ones,

383 while the EV items, words, were the most abstract stimuli. As to emotions, only HAPPINESS

384 items were significantly easier than the remaining ones, corroborating results from previous

385 research in emotion recognition and emotion understanding (Delgado, 2012, 2016; Russell,

386 1994; Suzuki, Hoshino \& Shigemasu, 2006). From the perception science field, it has been

387 suggested that the "happiness superiority effect" could have evolved due to the fact that happy

388 faces are communicatively less ambiguous than the remaining facial expressions of emotion

389 (Becker, Anderson, Mortensen, Neufeld, and Neel, 2011)

390 Thus, the three tests are ready to be used as components of a higher-level measurement

391 process (Newton \& Shaw, 2013). A promising application field is the assessment of EK as a

392 mediator of change in social competence, given that EK is consistently associated with various

393 social and behavioral outcomes in children and teenagers (Trentacosta, \& Fine, 2010) and EK

394 deficits are found in disorders such as alexithymia (Lumley, Neely, \& Burger, 2007). 
396 We want to thank the "Fundación Salamanca Ciudad de Cultura y Saberes" for lending us a quiet

397 space in the DA2 Domus Artium in order to get a heterogeneous participants' sample. 


\section{References}

400 Barrett, L. F. (2006). Solving the emotion paradox: Categorization and the experience of emotion. 401 Personality and Social Psychology Review, 10, 20-46.

402 Barrett, L.F. (2009a). The future of Psychology: Connecting mind to brain. Perspectives on 403 Psychological Science, 4, 326-339. doi:10.1111/j.1745-6924.2009.01134.x

404 Barrett, L.F. (2009b). Variety is the spice of life: A Psychologist Constructionist approach to 405 understanding variability in emotion. Cognition \& Emotion, 23, 1284-1306.

406 doi:10.1080/02699930902985894

407 Barrett, L.F. (2012). Emotions are real. Emotion, 12, 413-429. doi:10.1037/a0027555

408 Barrett, L.F. (2014). The conceptual act theory: a prècis. Emotion Review, 6, 292-297. 409 doi:10.1177/1754073914534479

410 Becker, D.V., Anderson, U.S., Mortensen, C.R., Neufeld, S.L., \& Neel, R. (2011). The face in 411 the crowd effect unconfounded: Happy faces, not angry faces, are more efficiently detected in 412 single- and multiple-target visual search tasks. Journal of Experimental Psychology: General, 413 140, 637-659. doi: 10.1037/a0024060

414 Coan, J.A., \& Gonzalez, M.Z. (2015). Emotions as emergent variables. In L.F. Barrett \& J.A. 415 Russell (Eds.), The psychological construction of emotion (pp. 209-226). New York: Guilford. 416 Delgado, A.R. (2007). Using the Rasch Model to quantify the causal effect of instructions. 417 Behavior Research Methods, 39, 570-573.

418 Delgado, A. (2012). Using the Rasch Model to test the psychometric quality of emotion 419 recognition items. Journal of the International Neuropsychological Society, 18 (S1), 69.

420 Delgado, A.R. (2016). Measuring Emotion Understanding with the Rasch Model. Actualidades 421 en Psicología, 30, 47-56. doi: http://dx.doi.org/10.15517/ap.v29i119.21516 
422 Engelhard, G., \& Wang, J. (2014). Alternative measurement paradigms for measuring executive

423 functions: SEM (formative and reflective models) and IRT (Rasch models). Measurement, 12,

424 102-108. doi: $10.1080 / 15366367.2014 .943592$

425 Hall, J.A., Gunnery, S.D., \& Horgan, T.G. (2016). Gender differences in interpersonal accuracy.

426 In J.A. Hall, M. S. Mast and T.V. West (eds.), The social psychology of perceiving others

427 accurately (pp. 309-327). Cambridge: Cambridge University Press.

428

429

430

431

432

433

434

435

436

437

438

439

440

441

442

443

Linacre, J.M. (2013). Winsteps Rasch measurement computer program, version 3.80.1. Chicago: Winsteps.com.

Lindquist, K.A., Gendron, M., Barrett, L. F., \& Dickerson, B.C. (2014). Emotion perception, but not affect perception, is impaired with semantic memory loss. Emotion, 14, 375-387. doi:

$10.1037 / \mathrm{a} 0035293$

Lindquist, K. A., Wager, T. D., Kober, H., Bliss-Moreau, E., \& Barrett, L. F. (2012). The brain basis of emotion: A meta-analytic review. Behavioral and Brain Sciences, 35, 121-143.

$$
10.1017 / \mathrm{S} 0140525 \mathrm{X} 11000446
$$

LiveCode 4.6 [computer software]. (2011). Retrieved from http://runrev.com/

Lumley, M. A., Neely, L. C., \& Burger, A. J. (2007). The assessment of alexithymia in medical settings: Implications for understanding and treating health problems. Journal of Personality Assessment, 89, 230-246. doi: 10.1080/00223890701629698

Luque-Reca, O., Augusto-Landa, J.M., \& Pulido-Martos, M. (2016). Emotional intelligence and depressive symptoms in Spanish institutionalized elders: does emotional self-efficacy act as a mediator? PeerJ, 4, e2246. doi: 10.7717/peerj.2246

MacCann, C., Joseph, D.L., Newman, D.A., \& Roberts, R.D. (2014). Emotional intelligence is a 
444 second stratum factor of intelligence: evidence from hierarchical and bifactor models.

$445 \quad$ Emotion, 14, 358-74. doi:10.1037/a0034755

446 Matthews, G., Zeidner, M., \& Roberts, R.D. (2012). Emotional intelligence: A promise

447 unfulfilled? Japanese Psychological Research, 54, 105-127. doi: 10.1111/j.1468-

$448 \quad 5884.2011 .00502 . x$

449 Michell, J. (1999). Measurement in psychology. Cambridge: Cambridge University Press.

450 Miguel, J.P., Silva, J.T., \& Prieto, G. (2013). Career Decision Self-Efficacy Scale - Short Form:

451 A Rasch analysis of the Portuguese version. Journal of Vocational Behavior, 82, 116-123.

$452 \quad$ doi.org/10.1016/j.jvb.2012.12.001

453 Molinari, C., Burin, D., Saux, G., Barreyro, J. P., Irrazabal, N., Bechis, M .S., ... Ramenzoni. V. 454 (2009). Fictional characters' emotional state representation: What is its degree of specificity? 455 Psicothema, 21, 9-14.

456 Newton, P.E., \& Shaw, S.D. (2013). Standards for talking and thinking about validity.

457 Psychological Methods, 18, 301-19. doi: 10.1037/a0032969

458 Orchard, B., MacCann, C., Schulze, R. Matthews, G., Zeidner, M., \& Roberts, R. D. (2009).

459 New Directions and Alternative Approaches to the Measurement of Emotional Intelligence.

460 In C. Stough, D.H. Saklofske, \& J. D. A. Parker, (Eds.), Advances in the Measurement of

$461 \quad$ Emotional Intelligence (pp. 321-344). New York: Springer.

462 Prieto, G., Delgado, A.R., Perea M.V., \& Ladera, V. (2010). Scoring Neuropsychological Tests

463 Using the Rasch Model: An Illustrative Example With the Rey-Osterreith Complex Figure.

464 The Clinical Neuropsychologist, 24, 45-56.

465 Rasch, G. (1960). Probabilistic models for some intelligence and attainment tests. Copenhagen, 466 Denmark: Danish Institute for Educational Research. 
467 Raz, G., Touroutoglou, A., Wilson-Mendenhall, C., Gilam, G., Lin, T. Gonen, T., ... ,\& Barrett, 468 L.F. (2016). Functional connectivity dynamics during film viewing reveal common networks 469 for different emotional experiences. Cognitive, Affective, \& Behavioral Neuroscience. doi: $470 \quad 10.3758 / \mathrm{s} 13415-016-0425-4$

471 Real Academia Española (2015). Corpus del Español del Siglo XXI, CORPES XXI. 472 [http://web.frl.es/CORPES/view/inicioExterno.view]

473 Reckase, M.D. (1979). Unifactor latent trait models applied to multifactor tests: results and 474 implications. Journal of Educational Statistics, 4, 207-230.

475 Rey, L., Extremera, N., \& Pena, M. (2016), Emotional competence relating to perceived stress 476 and burnout in Spanish teachers: a mediator model. PeerJ, 4, e2087. doi: 10.7717/peerj.2087 477 Russell, J. A. (1994). Is there universal recognition of emotion from facial expression? A review 478 of the cross-cultural studies. Psychological Bulletin, 115, 102-141.

479 Searle, J.R. (2010). Making the social world: The structure of human civilization. Oxford: $480 \quad$ Oxford University Press.

481 Soderberg C.K., Callahan, S.P., Kochersberger, A.O., Amit, E., \& Ledgerwood, A. (2015). The 482 effects of psychological distance on abstraction: Two meta-analyses. Psychological Bulletin, $483 \quad$ 141, 525-48. doi: 10.1037/bu10000005

484 Suzuki, A., Hoshino, T., \& Shigemasu, K. (2006). Measuring individual differences in 485 sensitivities to basic emotions in faces. Cognition, 99, 327-353

486 Thompson, A.E., \& Voyer, D. (2014). Sex differences in the ability to recognise non-verbal 487 displays of emotion: a meta-analysis. Cognition and Emotion, 28, 1164-1195. doi: $488 \quad 10.1080 / 02699931.2013 .875889$ 
489 Tracy, J. L., \& Randles, D. (2011). Four models of basic emotions: A review of Ekman and

490 Cordaro, Izard, Levenson, and Panksepp and Watt. Emotion Review, 3, 397-405. doi:

$491 \quad 10.1177 / 1754073911410747$

492 Trentacosta, C. J. \& Fine, S. E. (2010). Emotion knowledge, social competence, and behavior

493 problems in childhood and adolescence: A meta-analytic review. Social Development, 19, 1-

494 29. doi: $10.1111 / j .1467-9507.2009 .00543 . x$

495 Trope, Y., \& Liberman, N. (2010). Construal Level Theory of Psychological Distance.

496 Psychological Review, 117, 440-463. doi: 10.1037/a0018963

497 Wilson-Mendenhall, C. D., Barrett, L. F., Simmons, W. K., \& Barsalou, L. W. (2011).

498 Grounding emotion in situated conceptualization. Neuropsychologia, 49, 1105-1127. doi:

$499 \quad$ 10.1016/j.neuropsychologia.2010.12.032

500 Wu, M., Tam, H.P., \& Jen, T.H. (2017). Educational measurement for applied researchers:

501 Theory into practice. Singapore: Springer Nature. 